\title{
Exome sequencing and SNP analysis detect novel compound heterozygosity in fatty acid hydroxylase- associated neurodegeneration
}

\author{
Tyler Mark Pierson ${ }^{\star 1,2}$, Dimitre R Simeonov ${ }^{3}$, Murat Sincan ${ }^{3}$, David A Adams ${ }^{1,3}$, Thomas Markello ${ }^{1,4}$, \\ Gretchen Golas $^{4,5}$, Karin Fuentes-Fajardo ${ }^{1}$, Nancy F Hansen ${ }^{6}$, Praveen F Cherukuri ${ }^{6}$, Pedro Cruz ${ }^{6}$, \\ James C Mullikin for the NISC Comparative Sequencing Program ${ }^{6,7}$, Craig Blackstone ${ }^{2}$, Cynthia Tifft ${ }^{1,4}$, \\ Cornelius F Boerkoel $^{1}$ and William A Gahl ${ }^{1,4}$
}

Fatty acid hydroxylase-associated neurodegeneration due to fatty acid 2-hydroxylase deficiency presents with a wide range of phenotypes including spastic paraplegia, leukodystrophy, and/or brain iron deposition. All previously described families with this disorder were consanguineous, with homozygous mutations in the probands. We describe a 10-year-old male, from a non-consanguineous family, with progressive spastic paraplegia, dystonia, ataxia, and cognitive decline associated with a sural axonal neuropathy. The use of high-throughput sequencing techniques combined with SNP array analyses revealed a novel paternally derived missense mutation and an overlapping novel maternally derived $\sim 28$-kb genomic deletion in $F A 2 H$. This patient provides further insight into the consistent features of this disorder and expands our understanding of its phenotypic presentation. The presence of a sural nerve axonal neuropathy had not been previously associated with this disorder and so may extend the phenotype.

European Journal of Human Genetics (2012) 20, 476-479; doi:10.1038/ejhg.2011.222; published online 7 December 2011

Keywords: fatty acid 2-hydroxylase; fatty acid hydroxylase-associated neurodegeneration; exome sequencing; deletion analysis; neuropathy

\section{INTRODUCTION}

Fatty acid 2-hydroxylase (FA2H) is an NADPH-dependent monooxygenase that is essential for maintenance of the myelin sheath surrounding neuronal axons. This enzyme converts free fatty acids to 2-hydroxy fatty acids (hFAs), which are then incorporated into membrane sphingolipids (hFA-sphingolipids). ${ }^{1}$ These compounds have a specific temporal expression pattern and are not required in early development, but are essential as individuals mature. For example, fa2h-null mice develop normally structured myelin in the absence of hFA-sphingolipids; but as they mature, the lack of these compounds results in axonal dysfunction. ${ }^{2,3}$ Similar findings are seen in affected humans with FA2H deficiency, manifesting with several demyelinating phenotypes including spastic paraplegia type 35 (SPG35; MIM\#612319), 'leukodystrophy, dysmyelinating, and spastic paraparesis with or without dystonia' (MIM\#612443), or neurodegeneration with brain iron accumulation. ${ }^{4-8}$ These are collectively referred to as fatty acid hydroxylase-associated neurodegeneration (FAHN).

Phenotypic features of FAHN include the childhood onset of spasticity, dystonia, seizures, optic atrophy, cognitive decline, cerebellar atrophy, leukodystrophy, and brain iron accumulation. ${ }^{4-8}$ Several consanguineous families have been described with different combinations of these features, with the inconsistent features possibly reflecting the effects of homozygous mutations in other genes modifying the phenotypes. ${ }^{4-8}$ Alternatively, the amount of residual enzyme activity may be having a role, with individuals possessing a complete loss of FA2H activity having more severe neurodegeneration than individuals with residual FA2H activity. We describe an outbred individual with FAHN who possesses several typical clinical features of the disorder; however, he also has an axonal sensory neuropathy not previously associated with FAHN.

\section{METHODS}

SNP analysis and real-time PCR

The NHGRI Genomics Core lab performed SNP determinations using the Illumina Bead Array Platform (HumanOmniExpress, Illumina Corp., San Diego, CA, USA). Genome-wide fluorescent intensities and genotype calls were analyzed using Bead Studio and Genome Studio (Illumina Corp.). Analysis of copy-number variations was performed using PennCNV software, ${ }^{9}$ and visual inspection using Genome Studio version 2010v3 build36/hg18. ${ }^{10}$ The comparative $\mathrm{Ct}$ method was used to determine FA2H copy number by quantitative PCR (qPCR) using TaqMan primer-probe assays targeting exon 6 of FA2H (Hs00499334_cn) and a control gene LMNB1 (Hs02997647_cn; Applied Biosystems, Foster City, CA, USA).

\section{Exome analysis}

Solution hybridization exome capture was carried out at NISC using the SureSelect Human All-Exon System (Agilent Technologies, Santa Clara, CA, USA). ${ }^{11}$ The manufacturer's protocol version 1.0, compatible with Illumina

\footnotetext{
${ }^{1} \mathrm{NIH}$ Undiagnosed Diseases Program, NIH Office of Rare Diseases Research and NHGRI, Bethesda, MD, USA; ${ }^{2}$ Neurogenetics Branch, NINDS, Porter Neuroscience Research Center, Bethesda, MD, USA; ${ }^{3}$ Medical Genetics Branch, NHGRI, NIH, Bethesda, MD, USA; ${ }^{4}$ Office of the Clinical Director, NHGRI, NIH, Bethesda, MD, USA; ${ }^{5}$ Intramural Program of the Office of Rare Diseases Research, Office of the Director, NIH, Bethesda, MD, USA; ${ }^{6}$ Comparative Genomics Unit, Genome Technology Branch, NHGRI, NIH, Bethesda, MD, USA; ${ }^{7} \mathrm{NIH}$ Intramural Sequencing Center, NHGRI, NIH, Bethesda, MD, USA

${ }^{*}$ Correspondence: Dr TM Pierson, Neurogenetics Branch, NINDS, Porter Neuroscience Research Center, Building 35, Room 2A-1000, 35 Convent Drive, MSC 3705 , Bethesda, MD 20892-3705, USA. Tel: +1 301403 4231; Fax: +1 301496 7157; E-mail: piersonty@ninds.nih.gov
}

Received 18 July 2011; revised 28 September 2011; accepted 28 October 2011; published online 7 December 2011 
paired-end sequencing, was used with the exception that DNA fragment size and quality were measured with $2 \%$ agarose gels stained with Sybr Gold. Captured regions totaled $\sim 38 \mathrm{Mb}$. Flow cell preparation and 76-bp paired end read sequencing were performed at NISC per protocol of GAIIx sequencer (Illumina Corp.). Sequence alignment, genotype calling, and annotation were performed at NISC and are described elsewhere. ${ }^{12,13}$ Additional annotation and prioritization of genotypes according to disease-causing potential was performed using methods described earlier ${ }^{13}$ and with VAR-MD (Sincan M et al, unpublished data). The dbSNP132 database (that includes data from the 1000 genomes project) and a local database of 177 exomes were used to exclude common DNA variants.

\section{RESULTS}

\section{Clinical studies}

The proband (II-2) was born to healthy non-consanguineous parents, and has a healthy brother (II-1). There was no family history of neurological disease (Figure 1). At 10 years of age, he presented to the NIH Undiagnosed Diseases Program with a 7-year history of progressive neurodegeneration. The patient developed normally until age three, when he suffered a febrile illness and afterwards 'started tripping often'. Over the next 6 months he developed lower extremity spasticity, dystonic foot inversion, and poor balance. Symptoms evolved over the next few years to include dysarthria, neck weakness, and a 'hunched over' posture. He has not had seizures. He requires a walker to ambulate. Biopsies of skin, muscle, and sural nerve were reported to be normal.

Brain MRIs revealed progressive neurodegenerative changes. At 4 years, a punctate focus of T2 hyperintensity was noted in the right centrum semiovale, which a year later had evolved into numerous punctate foci in the periventricular and deep white matter. At 9 years, these changes developed into diffuse white matter signal abnormalities associated with cerebellar atrophy. A current study showed continued neurodegeneration with findings of a thin corpus callosum, brainstem/ cerebellar atrophy, and patchy white matter T2 signal abnormalities in the periventricular atrial regions consistent with demyelination (Figure 2). Borderline mineralization of the globi pallidi was noted.

On examination, the patient was awake and engaging. He answered questions and followed commands slowly. He had monotonous breathy speech but no language deficits. He had mask-like facies with some expressive capability. There were no retinal or optic nerve abnormalities. He had a stooped posture and neck weakness. His legs had diminished muscle bulk and strength, associated with hyperreflexia and spasticity. He had mild dysmetria and ataxia, but no obvious dystonic or choreiform movements. Sensation was normal.

Neuropsychological testing revealed a full scale IQ of 56 (verbal comprehension: 75, perceptual reasoning: 61, working memory: 68 , processing speed: 50 ; normal: $>70$ ). These results were significantly worse than 4 years prior, when he scored within the average range. Nerve conduction studies indicated sural SNAP responses had decreased amplitudes $(4 \mu \mathrm{V}$; normal: $>8 \mu \mathrm{V})$ and conduction velocity ( $35 \mathrm{~m} / \mathrm{s}$; normal: $>40 \mathrm{~m} / \mathrm{s}$ ) consistent with an axonal sensory neuropathy without demyelination.

\section{Mutation detection}

Evaluation of prioritized sequence variants derived from the proband's exome sequencing (ES) identified a novel, apparently homozygous, missense mutation in exon 5 of FA2H (c.707. T>C; p. F236S). The father was heterozygous for this mutation, but the mother was apparently homozygous for the wild-type reference sequence; however, with more detailed analysis and correlation with SNP microarray data, maternal hemizygosity was suspected. SNP microarrays revealed that the mother and the proband harbored a $\sim 28$-kb genomic deletion encompassing exons 3-7 that overlapped
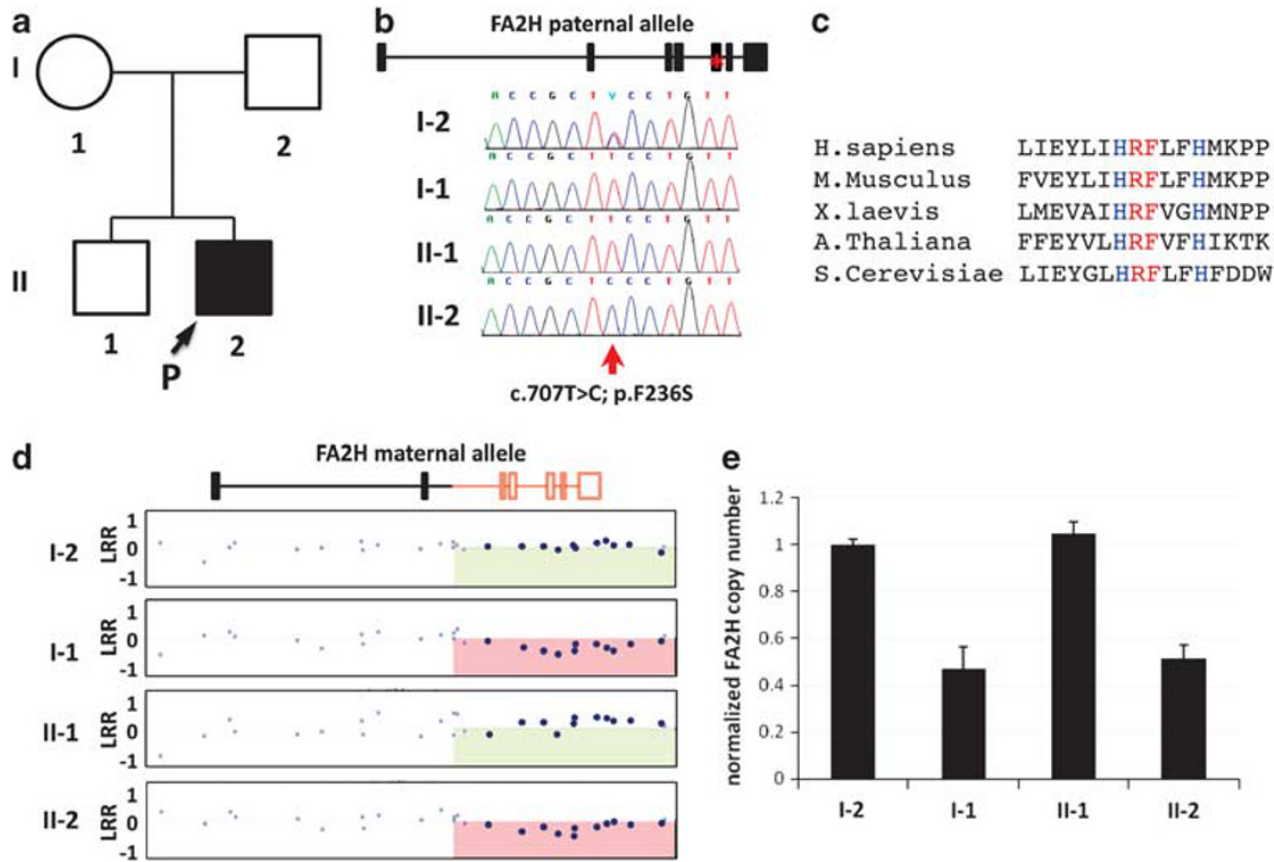

Figure 1 Pedigree and $F A 2 H$ mutations. (a) Family pedigree. The proband is indicated by an arrow. The affected individual is indicated in black. (b) Segregation of the paternally inherited $F A 2 H$ missense mutation (c.707C $>$ T; p.F236S). Schematic diagram of genomic locus and sequencing chromatogram (star and arrow represent mutation). (c) F236 and R235 are highly conserved residues across species. (d) Fluorescence intensity plot of SNP array showing a $\sim 28-\mathrm{kb}$ single copy deletion in proband and mother (FA2H exons 3-7). (e) qPCR results for FA2H exon 6 confirming the hemizygous deletion in the mother (I-1) and the proband (II-2). 

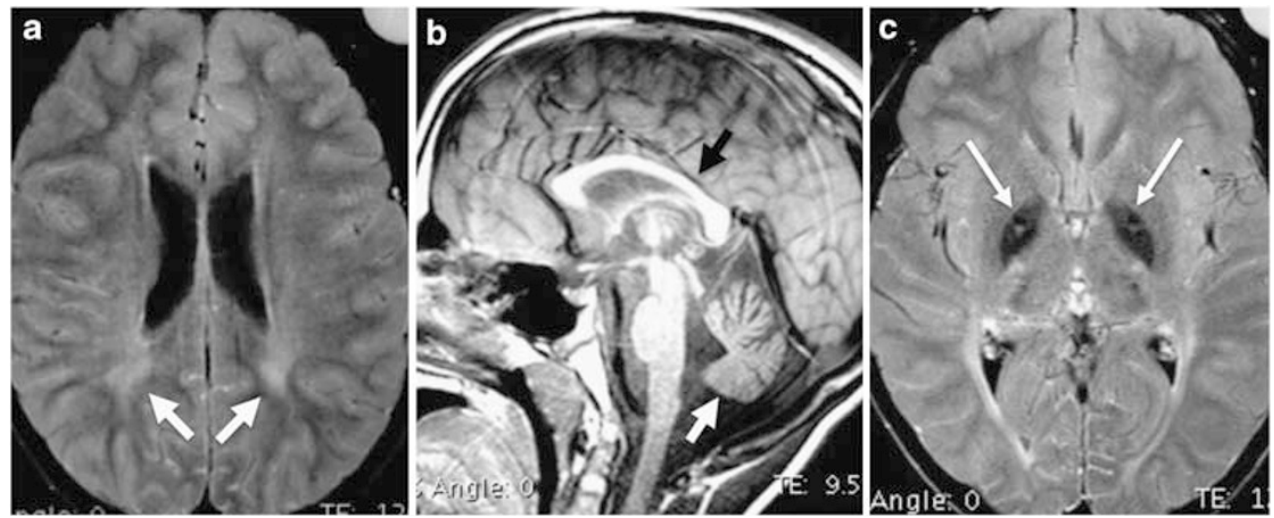

Figure 2 Brain MRI (3Tesla). (a) Diffuse white matter signal abnormalities consistent with demyelinating leukodystrophy (arrows; axial FLAIR). (b) Thin corpus callosum (black arrow) and mild brainstem/cerebellar atrophy (white arrow; sagittal T1-weighted). (c) Borderline mineralization of the globi pallidi (arrows; axial FLAIR).

the paternal mutation. Additional qPCR evaluation of exon 6 of FA2H confirmed the hemizygous deletion in the mother and the proband (Figure 2). Together, these data indicated that the patient was compound heterozygous for a missense mutation and deletion in $F A 2 H$ (Figure 1). The variant was seen once, in a heterozygous state, among 177 prior exomes sequenced at our facility. The dbSNP132 database contained no instances of the variant.

\section{DISCUSSION}

ES is a high-throughput genomic sequencing technique in which most of an individuals' exons are analyzed for genetic variants in order to determine pathogenic mutations. ${ }^{12,13}$ This information can be used in combination with other genomic evaluations to identify candidate genes for complex cases. We report a case where ES and SNP array analyses were used together along with phenotypic information to make a diagnosis of an extremely rare disorder. This result indicates that these methods are an efficient means to screen for the molecular bases of genetically heterogeneous disorders such as spastic paraplegias or leukodystrophies. Without these methods, the likelihood of making this rare diagnosis would have been dramatically decreased.

The maternally inherited $\sim 28$-kb genomic deletion encompasses sequences that had previously been shown to be essential for FA2H function. ${ }^{5,8}$ The paternally inherited mutation (c.707 T > C; p.F236S) alters a phenylalanine residue residing in an iron-binding histidine motif of the catalytic site. ${ }^{14}$ Of note, another family with a homozygous mutation affecting the adjacent amino acid (p.R235C) had enzyme activity that was decreased by $20-40 \%{ }^{4,6}$ Both F236 and R235 are highly conserved across genera including mice, the frog X. laevis, the plant A. thaliana, and the yeast $S$. cerevisiae (Figure 1), and thus are likely to be important for FA2H function.

Previously reported patients homozygous for putative null mutations or deletions had a severe FAHN disorder that additionally included dysphagia, optic atrophy, seizures, and severe cognitive decline. ${ }^{6,8}$ In contrast, patients homozygous for amino-acid substitutions (eg, p.R235C and p.D35Y) generally had milder phenotypes comprising only spastic paraplegia and mild cognitive decline. ${ }^{4-6}$ Our patient, with a combination of a null deletion and a missense substitution, displayed an intermediate phenotype.

This non-consanguineous family provides the first example where it is unlikely that homozygous mutations in other genes are confounding the phenotype. Our patient had many of the features of the previously described consanguineous families including spasticity, ataxia, dystonia, cognitive decline, and leukodystrophy, which support the association of these features with FA2H deficiency. However, there were some differences in our case from these cases and transgenic mouse models. Our patient did not have dysphagia, optic atrophy, or seizures at this time, and his cognition was not severely impaired. Continued monitoring will determine whether these features will develop later. Interestingly, this patient did have an axonal sensory neuropathy, a previously undescribed feature of FAHN. This finding is especially interesting as the etiology of FAHN is considered to involve myelin abnormalities and not direct axonal toxicity, although fa2h-null mice had late-onset peripheral demyelinating and axonal neuropathies. ${ }^{2,3}$ Determining whether this feature is a consistent finding in FAHN will require identification of additional patients, but may indicate a need to consider FAHN in children presenting with sensory neuropathy.

In summary, high-throughput genomic sequencing and SNP analysis allowed the focused evaluation of genes involved in spastic paraplegias and leukodystrophies, and were important for the diagnosis of a single family member with an extremely rare disorder. Further insight into this rare disorder was achieved by the evaluation of this non-consanguineous family, with the potential addition of sensory neuropathy to its phenotypic profile.

\section{CONFLICT OF INTEREST}

The authors declare no conflict of interest.

\section{ACKNOWLEDGEMENTS}

We are grateful to the patients and the parents of the family for their cooperation. We thank Shannon McNeil, Ronald Austin, Jose Salas, Chevalia Robinson, Joy Bryant, and Cheryl Hipple, Eva Baker, Bryan Brooks, Barrington Burnett, Kenneth Fischbeck, Roxanne Fischer, Hiroko Hama, Tanya Lehky, Joseph Snow, Gilbert Vezina, Lynne Wolfe, and Sandra Yang, for administrative, clinical and technical assistance, and critical analysis. This work was supported by the NIH Undiagnosed Diseases Program and the Intramural Research Program of the National Human Genome Research Institute, National Institutes of Health.

1 Hama H: Fatty acid 2-hydroxylation in mammalian sphingolipid biology. Biochim Biophys Acta 2010; 1801: 405-414.

2 Zöller I, Meixner M, Hartmann D et al: Absence of 2-hydroxylated sphingolipids is compatible with normal neural development but causes late-onset axon and myelin sheath degeneration. J Neurosci 2008; 28: 9741-9754. 
3 Potter KA, Kern MJ, Fullbright G et al: Central nervous system dysfunction in a mouse model of Fa2h deficiency. Glia 2011; 59: 1009-1021.

4 Dick KJ, Al-Mjeni R, Baskir W et al: A novel locus for an autosomal recessive hereditary spastic paraplegia. Neurology 2008; 22: 248-252.

5 Edvardson S, Hama H, Shaag A et al: Mutations in the fatty acid 2-hydroxilase gene are associated with leukodystrophy with spastic paraparesis and dystonia. Am J Hum Genet 2008; 83: 643-648.

6 Dick KJ, Eckhardt M, Paisán-Ruiz $\mathrm{C}$ et al: Mutation of FA2H underlies a complicated form of hereditary spastic paraplegia (SPG35). Hum Mutat 2010; 31: E1251-E1260.

7 Kruer MC, Paisán-Ruiz C, Boddaert N et al: Defective FA2H leads to a novel form of neurodegeneration with brain iron accumulation (NBIA). Ann Neurol 2010; 68: 611-618.

8 Garone C, Pippucci T, Cordelli DM et al: FA2H-related disorders: a novel c. $270+3 \mathrm{~A}>\mathrm{T}$ splice-site mutation leads to a complex neurodegenerative phenotype. Dev Med Child Neurol 2011; 11: 1469-8749.
9 Wang K, Li M, Hadley D et al: PennCNV: An integrated hidden Markov model designed for high-resolution copy number variation detection in whole-genome SNP genotyping data. Genome Res 2007; 17: 1665-1674.

10 Manoli I, Golas G, Westbroek W et al: Chediak-Higashi syndrome with early developmental delay resulting from paternal heterodisomy of chromosome 1. Am J Med Genet 2010; 152A: 1474-1483.

11 Gnirke A, Melnikov A, Maguire J et al: Solution hybrid selection with ultra-long oligonucleotides for massively parallel targeted sequencing. Nat Biotechnol 2009; 27: 182-189.

12 Teer JK, Bonnycastle LL, Chines PS et al: Systematic comparison of three genomic enrichment methods for massively parallel DNA sequencing. Genome Res 2010; 20: 1420-1431.

13 Johnston JJ, Teer JK, Cherukuri PF et al: Massively parallel sequencing of exons on the $\mathrm{X}$ chromosome identifies RBM10 as the gene that causes a syndromic form of cleft palate. Am J Hum Genet 2010; 86: 743-748.

14 Alderson NL, Rembiesa BM, Walla MD, Bielawska A, Bielawski J, Hama H: The human FA2H gene encodes a fatty acid 2-hydroxylase. J Biol Chem 2004; 279: 48562-48568. 